\title{
Lie Cebiroidleri Üzerindeki Lagrange Dinamiğinin Eşlenmesi Problemi Üzerine
}

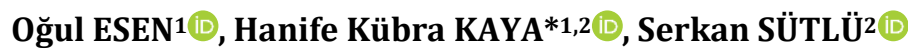 \\ ${ }^{1}$ Gebze Teknik Üniversitesi, Temel Bilimler Fakültesi, Matematik Bölümü, 41400, Kocaeli, Türkiye \\ ${ }^{2}$ Işılk Üniversitesi, Fen Edebiyat Fakültesi, Matematik Bölümü, 34980, İstanbul, Türkiye
}

(Alınış / Received: 19.10.2020, Kabul / Accepted: 02.04.2021, Online Yayınlanma / Published Online: 15.08.2021)

\section{Anahtar Kelimeler}

Lie grupoidi,

Lie cebiroidi,

Euler-Lagrange denklemleri
Özet: Lie cebiroidleri, bir anlamda tanjant demetini ve Lie cebiri yapısını beraber ihtiva eden ve fakat daha genel olan geometrik inşaalardır. Lagrange dinamiğinin en genel ifadesi Lie cebiroidleri üzerinde mümkündür. Bu makalede, karşllıklı (Lie cebiroidi üzerinde tanımlı) etki içindeki iki Lagrange dinamiğinin beraber davranışı, geometrik ve cebirsel bir yol ile elde edilecektir. Bu bakış açısı ile etkileşim, Lie cebiroidlerinin birbirleri üzerine olan lineer temsilleri (etkileri) ifade edilecektir. Böylece, belirli uyumluluk şartını sağlayan karşılıklı etki içindeki iki Lie cebiroidinin eşlenmesi, diğer bir ifade ile tek bir Lie cebiroidi olarak yazılması sağlanacaktır. Sonrasında ise eşlenmiş Lie cebiroidi üzerinde Lagrange dinamiği yazılacaktır. Elde edilecek kollektif (eşlenmiş) hareket denklemleri, bireysel davranışların gözlemlenmesinin yanı sıra karşılıklı etki terimlerinin de belirlenmesine olanak verecektir. Çalışmamız esnasında bir çok örnek sunularak teorik tanımların daha net anlatımı yakalanmaya çalışılacaktır.

\section{On The Problem of Matched Lagrangian Dynamics on Lie Algebroids}

\section{Keywords}

Lie groupoid,

Lie algebroid,

Euler-Lagrange equations

\begin{abstract}
Lie algebroids are geometric constructions generalizing both tangent bundles and Lie algebras. Lagrangian dynamics is possible on Lie algebroid frameworks in its most general form. In this work, we obtain the joint behaviour of two mutually interacting Lagrangian systems in a geometric and an algebraic way. Here, the interaction is decoded into linear representations (actions) of two Lie algebroids onto each other. By this means, mutally interacting two Lie algebroids those satisfying some certain compatibility condition are matched, in other words, they are recast as trivially intersecting Lie subalgebroids of a single Lie algebroid. Then, Lagrangian dynamics is recast on the matched Lie algebroid. In this framework, the equations involve both the dynamics of constitutive subsystems and the action terms. Along with the theory, we provide several examples.
\end{abstract}

\section{Giriş}

Klasik anlamda, konfigürasyon uzayı bir $M$ katmanı olan bir fiziksel sistem için hareketi belirleyen EulerLagrange denklemleri, tanjant demeti TM üzerinde tanımlı bir Lagrange fonksiyonu $L$ aracılığıla belirlenir [1, 2]. Eğer $M$ üzerindeki yerel koordinatları $\left(q^{i}\right), T M$ üzerindeki koordinatları $\left(q^{i}, \dot{q}^{i}\right)$ ile gösterirsek etki integrali $\int L d t$ için sabit sınır koşullarını sağlayan eğriler üzerinde varyasyon alınarak ve Hamilton prensibi uygulanarak EulerLagrange denklemlerine

$$
\frac{d}{d t}\left(\frac{\partial L}{\partial \dot{q}^{i}}\right)-\frac{\partial L}{\partial q^{i}}=0
$$

ulaşılır.
Diğer yandan, eğer fiziksel sistemin konfigürasyon uzayı bir Lie grubu $G$ ise, tanjant demeti $T G$ de bir Lie grubu olacaktır [3]. Burada, eğer TG üzerinde tanımlı Lagrange fonksiyonu $G$ grubunun $T G$ üzerine sol etkisi altında değişmiyorsa, diğer bir ifade ile $G$ grubu $L$ Lagrange fonskiyonu için bir simetri ise, Lagrange indirgeme teoremi uygulanır [2, 4]. Bu da (Lie grubu $G^{\prime}$ nin) Lie cebiri $g$ üzerinde tanımlı indirgenmiş bir Lagrange fonksiyonu $l$ ve yine cebir üzerinde tanımlı Euler-Poincaré denklemlerini

$$
\frac{d}{d t}\left(\frac{\delta l}{\delta \xi}\right)-a d_{\xi}^{*}\left(\frac{\delta l}{\delta \xi}\right)=0
$$

verir. Burada, $\xi$ Lie cebirinin bir elemanı, $\delta l / \delta \xi$ Lagrange fonksiyonunun Fréchet türevi, $a d^{*}$ ise Lie cebiri $g^{\prime}$ nin dual uzayı $\mathrm{g}^{*}$ üzerinde adjoint temsilidir. 
Güncel sorulardan biri Euler-Lagrange denklemleri (1) ve Euler-Poincaré denklemleri (2)'nin tek bir denklemin özel durumu olarak yazılıp yazılamayacağı sorusu idi. $\mathrm{Bu}$, Lie cebiroidi üzerinde Lagrange dinamiği yazılarak 90’ların sonunda çözüldü [5]. Bu yaklaşım daha geometrik analizi, Lie cebiroidi üzerindeki tensör alanlarının marifetiyle yazılması $[6,7]$ 'de verildi.

Bu makalede ilgilendiğimiz soru ise bahsettiğimiz ilk temel üzerinde kurulu ve fakat şu şekildedir: İki fiziksel sistem ele alalım. Bunlar karşılıklı etki tepki içinde olsunlar. Yani beraber hareketleri esnasında bireysel hareketlerini sürdüremesinler. Bu durumda kollektif hareketi belirleyen Lagrange dinamik denklemleri de bireysel denklemlerin üst üste yazılması ile belirlenemez. Hareketi kontrol eden diferansiyel denklemlerde karşıllklı etki tepkiyi işaret eden terimler gözükecektir. Bu problem tek taraflı etki için yarı çarpım teorisi olarak ifade edimiş ve klasik mekanikten [8], akışkanlar ve plazma teorisine [9] kadar çok geniş uygulama alanı bulmuştur. Karşılıklı etki tepki içindeki iki Lagrange sisteminin kollektif davranışı, [10] çalışmasında iki EulerPoincaré denkleminin eşlenmesi ile başarılmıştır. Bu makalede eşlenmiş Lie cebiroidleri üzerinde Lagrange denklemleri yazılarak en genel şekilde Lagrange denklemlerinin eşlenmesi hedeflenmiştir.

$\mathrm{Bu}$ çalışma iki ana bölümden oluşmaktadır. Birinci bölümde Lie groupoidi ve Lie cebiroidi formal olarak tanıtılmış, Lagrange denklemleri yazılmıştır. İkinci bölümde, sırasıyla, karşılıklı etki tepki içindeki iki Lie groupoidinin ve iki Lie cebiroidinin eşlenmesi yapılmıştır. Eşlenmiş geometriler üzerinde ise etki tepki içindeki iki Lagrange sisteminin geometrik/cebirsel olarak eşlenmesi başarılmıştır. Teorik kavramlar bir çok örnek eşliğinde sunularak tartışma zenginleştirilmeye çalışılmıştır.

\section{Lie Cebiroidleri Üzerindeki Lagrange Dinamiği}

\subsection{Lie grupoidi ve Lie cebiroidi}

Lie grupoidi: $\mathcal{G}$ ve $B$ katmanları arasında

$$
\mathcal{G} \stackrel{\alpha}{\rightarrow} B, \quad \mathcal{G} \stackrel{\beta}{\rightarrow} B
$$

daldırma fonksiyonlarını düşünelim. Bu fonksiyonlara, sırasıyla, kaynak ve hedef fonksiyonları diye sesleneceğiz. Bunlara ilave olarak, bir de (nesne) gömme fonksiyonunun

$$
\varepsilon: B \rightarrow \mathcal{G}, \quad b \mapsto \tilde{b}
$$

varlığını farz edelim. $\mathcal{G} \times \mathcal{G}$ kartezyen çarpım uzayının

$$
\mathcal{G} * \mathcal{G}:=\left\{\left(g, g^{\prime}\right) \in \mathcal{G} \times \mathcal{G} \mid \beta(g)=\alpha\left(g^{\prime}\right)\right\}
$$

alt kümesine çarpılabilir elemanlar kümesi denir. Bu küme üzerindeki kısmi çarpma işlemi

$$
\mathcal{G} * \mathcal{G} \rightarrow \mathcal{G}, \quad\left(g, g^{\prime}\right) \mapsto g g^{\prime}
$$

ile gösterilir. İşte bu kısmi çarpma işlemi ile donatılan, her $\left(g, g^{\prime}\right),\left(g^{\prime}, g^{\prime \prime}\right) \in \mathcal{G} * \mathcal{G}, b \in B$, ve $g \in \mathcal{G}$ için

$$
\begin{aligned}
& \alpha\left(g g^{\prime}\right)=\alpha(g), \beta\left(g g^{\prime}\right)=\beta\left(g^{\prime}\right), \\
& g\left(g^{\prime} g^{\prime \prime}\right)=\left(g g^{\prime}\right) g^{\prime \prime}, \\
& \alpha(\tilde{b})=\beta(\tilde{b})=b, \\
& g \widetilde{\beta(g)}=g=\widetilde{\alpha(g)} g, \\
& \text { her } g \in \mathcal{G} \text { için } \alpha\left(g^{-1}\right)=\beta(g), \beta\left(g^{-1}\right)=\alpha(g)
\end{aligned}
$$

özelliklerini sağlayan $(\mathcal{G}, B, \alpha, \beta, \varepsilon)$ beşlisine Lie grupoidi denir, burada ters eleman $g^{-1} \in \mathcal{G}$ için $g^{-1} g=\widetilde{\beta(g)}, \quad g g^{-1}=\widetilde{\alpha(g)} \quad$ şeklindedir. $\quad B$ katmanının elemanları nesneler, $\mathcal{G}^{\prime}$ nin elemanları ise oklar, ya da morfizmalar olarak anılır. $B$ tabanı üzerindeki böyle bir $\mathcal{G}$ Lie grupoidi $\mathcal{G} \rightrightarrows B$, ya da kısaca $\mathcal{G}$ ile gösterilir.

Örnek 2.1: $M$ bir katman ve $G$ bir Lie grubu olsun. $M \times G \rightarrow M$ türevlenebilir sağ etkisini göz önünde bulunduralım. Bu etki sayesinde $M \times G \rightrightarrows M$ olarak göstereceğimiz bir Lie grupoidi yapısı elde edilir. $\mathrm{Bu}$ grupoidine $M$ üzerinde etki Lie grupoidi denir. Kaynak ve hedef fonksiyonları ile gömme fonksiyonu

$$
\begin{array}{ll}
\alpha: M \times G \rightarrow M, & \alpha(m, g):=m, \\
\beta: M \times G \rightarrow M, & \beta(m, g):=m g, \\
\varepsilon: M \rightarrow M \times G, & \varepsilon(m):=(m, e) .
\end{array}
$$

ile verilir. Kısmi çarpım ise, $m g=m^{\prime}$ olduğunda,

$$
(m, g) \cdot\left(m^{\prime}, g^{\prime}\right):=\left(m, g g^{\prime}\right)
$$

ile yazılır [11].

Örnek 2.2: $M$ katmanı üzerinde kartezyen çarpım uzayı $M \times M, M$ üzerinde bir Lie grupoidi yapısıyla donatılabilir ve bu oluşan Lie grupoidi, $M \times M \rightrightarrows M$ ikili grupoidi olarak adlandırılır. İkili grupoidinin kaynak ve hedef fonksiyonları ile gömme fonksiyonu şu şekildedir:

$$
\begin{array}{ll}
\alpha: M \times M \rightarrow M, & \alpha\left(m, m^{\prime}\right):=m, \\
\beta: M \times M \rightarrow M, & \beta\left(m, m^{\prime}\right):=m^{\prime}, \\
\varepsilon: M \rightarrow M \times M, & \varepsilon(m):=(m, m) .
\end{array}
$$

Kısmi çarpımı $m^{\prime}=n$ ise,

$$
\left(m, m^{\prime}\right) \cdot\left(n, n^{\prime}\right):=\left(m, n^{\prime}\right)
$$

şeklindedir [11].

Örnek 2.3: Yine, $M$ bir katman ve $G$ bir Lie grubu için, $M$ üzerinde üçlü Kartezyen çarpım uzayı $M \times$ $G \times M$ bir Lie grupoidi yapısı olarak düşünülebilir. $\mathrm{Bu}$ grupoide ise aşikar grupoidi adı verilir ve $M \times G \times$ $M \rightrightarrows M$ notasyonu ile gösterilir. Bu Lie grupoidinin kaynak, hedef ve gömme fonksiyonları 
$\alpha: M \times G \times M \rightarrow M, \quad \alpha\left(m, g, m^{\prime}\right):=m$,

$\beta: M \times G \times M \rightarrow M, \quad \beta\left(m, g, m^{\prime}\right):=m^{\prime}$,

$\varepsilon: M \rightarrow M \times G \times M, \quad \varepsilon(m)=\widetilde{m}:=(m, e, m)$

şeklinde olup kısmi çarpım ise, $m^{\prime}=n$ olduğunda,

$$
\left(m, g, m^{\prime}\right) \cdot\left(n, g^{\prime}, n^{\prime}\right):=\left(m, g g^{\prime}, n^{\prime}\right)
$$

olacaktır [11].

Lie cebiroidi: $\operatorname{Bir} M$ katmanı üzerinde $\operatorname{bir} \mathcal{A}$ Lie cebiroidi bir $\tau_{\mathcal{A}}: \mathcal{A} \rightarrow M$ vektör demeti olup üzerinde her $X, Y \in \Gamma(\mathcal{A})$, ve $M$ üzerinde tanımlı her $f$ fonksiyonu için

$$
[X, f Y]_{\mathcal{A}}=f[X, Y]_{\mathcal{A}}+\mathcal{L}_{a_{\mathcal{A}}(X)}(f) Y
$$

eşitliğini sağlayan ve çapa adı verilen bir $a_{\mathcal{A}}: \mathcal{A} \rightarrow$ $T M$ dönüşümü vardır. Burada çerçeve işlemi $[\bullet, \bullet]_{\mathcal{A}}$ kesitler uzayı $\Gamma(\mathcal{A})$ üzerinde (iki-lineer, antisimetrik, ve Jacobi özdeşliğini sağlayan) bir Lie çerçevesi olup, $\mathcal{L}_{a_{\mathcal{A}}(X)}(f)$ ile $f$ fonksiyonunun $a_{\mathcal{A}}(X)$ vektörü doğrultusundaki yönlü türevini gösterilmektedir. Çapa fonksiyonunun kesitler uzayına kısitlanması $a_{\mathcal{A}}: \Gamma(\mathcal{A}) \rightarrow \Gamma(T M)$ bir $C^{\infty}(M)$ modül homomorfizmasıdır. Yani, her $X, Y \in \Gamma(\mathcal{A})$ için

$$
a_{\mathcal{A}}\left([X, Y]_{\mathcal{A}}\right)=\left[a_{\mathcal{A}}(X), a_{\mathcal{A}}(Y)\right]
$$

eşitliği vardır. Bir Lie cebiroidi $\left(\mathcal{A}, \tau_{\mathcal{A}}, M, a_{\mathcal{A}},[\bullet, \bullet]_{\mathcal{A}}\right)$ beşlisi ile belirlenir [6, 12-14].

$M$ üzerinde bir yerel koordinat sistemi $\left(x^{i}\right), \mathcal{A}$ üzerinde bir yerel koordinat sistemi $\left(x^{i}, y^{\alpha}\right)$ olsun. Bunlara paralel olarak $\mathcal{A}$ 'nın kesit uzayı $\Gamma(\mathcal{A})$ için $\left(e_{\alpha}\right)$ baz takımını seçelim.

Bu durumda sırasıyla çapa dönüşümü $a_{\mathcal{A}}$ 'nın matris gösterimini ve Lie cebiroidi çerçevesi için yapı sabitlerini şu şekilde elde ederiz:

$$
a_{\mathcal{A}}\left(e_{\alpha}\right)=\left(a_{\mathcal{A}}\right)_{\alpha}^{i} \frac{\partial}{\partial x^{i}}, \quad\left[e_{\alpha}, e_{\beta}\right]_{\mathcal{A}}=C_{\alpha \beta}^{\gamma} e_{\gamma} .
$$

Bir Lie grupoidinin Lie cebiroidi: Nasıl türev alınarak bir Lie grubuna karşı gelen bir Lie cebiri var ise, bir Lie grupoidine karşı gelen Lie cebiroidi de mevcuttur. Şimdi bu durumu [14-17] kaynakları ışı̆̆ında inceleyelim. $(\mathcal{G}, B, \alpha, \beta, \varepsilon)$ bir Lie grupoidi olsun. Kaynak fonksiyonu $\alpha: \mathcal{G} \rightarrow B$ ve $g \in \mathcal{G}$ noktasinda tanjantı $T_{g} \alpha: T_{g} \mathcal{G} \rightarrow T_{\alpha(g)} B$ olsun. $\mathcal{G}$ Lie grupoidi ile ilişkilendirilen Lie cebiroidi iplikleri

$$
\mathcal{A}_{b} \mathcal{G}:=\operatorname{ker}_{\varepsilon(b)} \alpha,
$$

şeklindedir. $\mathrm{Bu}$ sayede $(\mathcal{A G}, \tau, B)$ vektör demeti tanımlanır. Bir başka deyişle $\mathcal{A G}, \mathcal{G}$ üzerinde $\alpha: \mathcal{G} \rightarrow B$ fonksiyonuna göre alınan dikey demetine karşılık gelir. $\tau: \mathcal{A G} \rightarrow B$ vektör demetinin kesitlerini $X \in$ $\Gamma(\mathcal{A G})$ ile gösterelim. Çapa fonksiyonu $a: \mathcal{A G} \rightarrow T B$ ise

$$
a(X(b))=T_{\tilde{b}} \beta \circ X(b)
$$

ile verilir, burada $T_{\tilde{b}} \beta: T_{\tilde{b}} \mathcal{G} \rightarrow T_{b} B$ ile $\beta: \mathcal{G} \rightarrow B$ hedef fonksiyonunun $\tilde{b}=\varepsilon(b) \in \mathcal{G}$ noktasındaki tanjantıdır. $\mathcal{A G} \rightarrow B$ kesitleri üzerindeki Lie çerçevesi $[\bullet, \bullet]_{\mathcal{A G}}$, grupoidi üzerindeki sol (veya sağ) vektör alanlarının Jacobi-Lie çerçevesi yardımıyla tanımlanır. Bir $X \in$ $\Gamma(\mathcal{A G})$ için, ona karşılık gelen bir sol değişmez vektör alanı $\overleftarrow{X} \in \Gamma(T \mathcal{G})$ vardır ve

$$
\overleftarrow{X}(g):=T_{\widetilde{\beta(g)}} \ell_{g} X(\beta(g))
$$

ile tanımlanır. $\mathrm{Bu}$ sayede Lie çerçeve işlemi, $X, Y \in$ $\Gamma(\mathcal{A G})$ kesitleri için

$$
[X, Y]_{\mathcal{A G}}(b):=[\overleftarrow{X}, \overleftarrow{Y}](\tilde{b})
$$

şeklinde yazılır, burada sağ tarafta verilen Jacobi-Lie çerçeve işlemidir. Öte yandan bir $X \in \Gamma(\mathcal{A G})$ için, ona karşılık gelen bir sağ değișmez vektör alanı $\vec{X} \in$ $\Gamma(T \mathcal{G})$ ise

$$
\vec{X}(g):=-T_{\overline{\alpha(g)}} r_{g} \circ T_{\overline{\alpha(g)}} \mathrm{i} n v(X(\alpha(g)),
$$

ile tanımlanır, burada inv: $\mathcal{G} \rightarrow \mathcal{G}$ tersine çevirme ve $T_{\widetilde{\alpha(g)}} r_{g}: T_{\widetilde{\alpha(g)}} \mathcal{G} \rightarrow T_{g} \mathcal{G} \quad$ ise $\quad \varepsilon(\alpha(g))=\widetilde{\alpha(g)} \in \mathcal{G}$ noktasinda $g \in \mathcal{G}$ ile verilen sağ ötelemenin tanjantıdır. Çerçeve işlemi $X, Y \in \Gamma(\mathcal{A} \mathcal{G})$ kesitleri için

$$
\overrightarrow{[X, Y]}_{\mathcal{A G}}=-[\vec{X}, \vec{Y}],
$$

şeklinde yazılır [11].

Örnek 2.4: Tanjant demetini, $\left(T M, \tau_{M}, M\right)$ üçlüsünü, düșünelim. $\mathrm{Bu}$ demet Jacobi-Lie çerçevesi ile donaltılmış ve çapa fonksiyonu $i d_{T M}$ olarak düşünüldüğünde bir Lie cebiroidi yapısına kavuşur. Tanjant demeti, Örnek (2.2) ile verilen $M \times M \rightrightarrows M$ ikili Lie grupoidinin cebiroidi olarak düşünülebilir [11].

Örnek 2.5: Bir Lie grubu $G,\{p\}$ ile göstereceğimiz tek bir nokta üzerinde Lie grupoidi yapısına sahip olacağı açıktır. Bu grupoidi özelinde her elemanın bir biri ile çarpılabildiğine dikkat edelim. $G \rightrightarrows\{p\}$ grupoidine karşı gelen Lie cebiroidi ise $g$ Lie cebirinin aynı tek nokta üzerindeki demet yapısıdır. Lie cebir çerçevesi Lie cebiroidi çerçevesi olacak, çapa fonksiyonu ise (tek nokta $M=\{p\}$ katmanının tanjant demeti $T M=$ $0_{p}$ olacağından) sıfır fonksiyonu olacaktır.

Bir başka örneğimize geçmeden önce bir $g$ Lie cebirinin sol etkisinin nasıl verildiğine bakalım. $g$ bir sonlu boyutlu reel bir Lie cebir olsun ve $\Phi: \mathfrak{g} \rightarrow$ $\Gamma(T M)$ ile tanımlanan $g$ Lie cebirinin $M$ katmanı üzerindeki bir sol etkisi, her $\xi, \varsigma \in \mathfrak{g}$ için,

$$
\Phi\left([\xi, \varsigma]_{\mathrm{g}}\right)=[\Phi(\xi), \Phi(\varsigma)]
$$

özelliğini sağlayan bir $\mathbb{R}$-lineer fonksiyondur, burada sağ taraftaki çerçeve, Jacobi-Lie çerçevesidir [18]. 
Örnek 2.6: Bir g Lie cebirinin bir sol etkisine olanak tanıyan bir $M$ katmanı alalım. Lie çerçeve işlemini koruyan böyle bir $g \rightarrow \Gamma(T M), \xi \mapsto X_{\xi}$ lineer fonksiyon vardır. Birinci bileşene göre izdüşüm olan aşikar demet $M \times \mathfrak{g} \rightarrow M$ üzerinde $m \in M$ ve $\xi \in \mathfrak{g}$ için, $a: M \times \mathfrak{g} \rightarrow T M, a(m, \xi):=X_{\xi}(m)$ ve çerçeve işlemi

$$
\begin{aligned}
& {[u, v]_{(M \times \mathfrak{g})}(m):=[u(m), v(m)]_{\mathfrak{g}}} \\
& \quad+\left(\mathcal{L}_{X_{u(m)}} v\right)(m)-\left(\mathcal{L}_{X_{v(m)}} u\right)(m)
\end{aligned}
$$

şeklinde verilir. Burada $u, v: M \rightarrow \mathfrak{g}$ fonksiyonları ile $(M \times \mathrm{g})$ așikar demetinin kesitleri, $\mathcal{L}_{X}(w)$ ile $X \in$ $\Gamma(T M)$ vektör alanı boyunca bir Lie cebir değerli $w: M \rightarrow \mathfrak{g}$ fonksiyonunun Lie türevi ve $[\bullet, \bullet]_{\mathfrak{g}}$ ile $\mathfrak{g}$ üzerindeki Lie çerçeve işlemi gösterilmiştir. Bu Lie cebiroidine etki Lie cebiroidi veya dönüşüm Lie cebiroidi denir. Bu Lie cebiroidi Örnek (2.1) ile verilen $M \times G \rightrightarrows M$ etki Lie grupoidine karşı gelen Lie cebiroididir [11].

\subsection{Lie cebiroidi üzerindeki Lagrange dinamiği}

$\mathrm{Bu}$ bölümde ise [5]'i takip ederek, Lie cebiroidi üzerindeki Lagrange mekaniğinin Lie cebiroidinin duali yardımıyla nasıl tanımlandığını yazalım. Öncelikle; $\mathcal{A}$ bir Lie cebiroidi, $\mathcal{A}^{*}$ da onun duali (vektör demeti olarak duali) olsun. Yukarıda, $\mathcal{A}$ Lie cebiroidinin yerel koordinat takımını $\left(x^{i}, y^{\alpha}\right)$ diye yazmıştık. Bu durumda, dual vektör demeti $\mathcal{A}^{*}$ üzerindeki bir yerel koordinat takımını da $\left(x^{i}, \mu_{\alpha}\right)$ diye gösterelim.

Öte yandan, $a_{\mathcal{A}}: \mathcal{A} \rightarrow T M$ çapa fonksiyonunun da katılımiyla

$$
a_{\mathcal{A}}\left(e_{\alpha}\right)=\left(a_{\mathcal{A}}\right)_{\alpha}^{i} \frac{\partial}{\partial x^{i}}, \quad\left[e_{\alpha}, e_{\beta}\right]=C_{\alpha \beta}^{\gamma} e_{\gamma}
$$

eşitliklerinden doğan $\quad\left(a_{\mathcal{A}}\right)_{\alpha}^{i}, C_{\alpha \beta}^{\gamma} \in C^{\infty}(M)$ foksiyonlarını da hatırlayalım. İspatı için okuyucularımızı [5] nolu çalışmaya yönlendireceğimiz aşağıdaki ilke teoremimizi verelim

Teorem 2.1: $\mathcal{A}$ bir Lie cebiroidi olmak üzere, dual vektör demeti $\mathcal{A}^{*}$ üzerinde doğal bir Poisson yapısı vardır. Bu yapı Denklem (21)'de verilen yerel koordinatlar cinsinden

$$
\begin{aligned}
& \left\{x^{i}, x^{j}\right\}=0, \\
& \left\{\mu_{\alpha}, \mu_{\beta}\right\}=C_{\alpha \beta}^{\gamma} \mu_{\gamma}, \\
& \left\{x^{i}, \mu_{\alpha}\right\}=\left(a_{\mathcal{A}}\right)_{\alpha}^{i}
\end{aligned}
$$

ile verilir.

Bir $L \in C^{\infty}(\mathcal{A})$ Lagrange fonksiyonunun (yerel koordinat takımı ile $L\left(x^{i}, y^{\alpha}\right)$ ) belirlediği enerji fonksiyonunu

$$
E_{L}:=\frac{\partial L}{\partial y^{\alpha}} y^{\alpha}-L
$$

ve Legendre dönüşümünü,

$$
\mathcal{A} \rightarrow \mathcal{A}^{*}, \quad\left(x^{i}, y^{\alpha}\right) \mapsto\left(x^{i}, \mu_{\alpha}=\frac{\partial L}{\partial y^{\alpha}}\right)
$$

tanımlayalım. Eğer Lagrange fonksiyonu (regüler) düzgün ise, yani Legendre dönüşümü (yerel) bir difeomorfizma ise, $\mathcal{A}^{*}$ üzerindeki Poisson yapısını $\mathcal{A}^{\prime}$ ya çekmek mümkündür. $\mathcal{A}$ üzerinde bu şekilde elde edilen Poisson yapısına Lagrange-Poisson yapısı denilir, ve Poisson çerçevesi

$$
\begin{aligned}
& \left\{x^{i}, x^{j}\right\}=0, \\
& \left\{\frac{\partial L}{\partial y^{\alpha}}, \frac{\partial L}{\partial y^{\beta}}\right\}=C_{\alpha \beta}^{\gamma} \frac{\partial L}{\partial y^{\gamma}}, \\
& \left\{x^{i}, \frac{\partial L}{\partial y^{\alpha}}\right\}=\left(a_{\mathcal{A}}\right)_{\alpha}^{i}
\end{aligned}
$$

ile belirlidir.

İşte $\mathcal{A}$ üzerinde, seçilen $L \in C^{\infty}(\mathcal{A})$ Lagrange fonksiyonunun tanımladığı Euler-Lagrange denklemleri de, yukarıdaki Lagrange-Poisson çerçevesi marifetiyle

$$
\frac{d x^{i}}{d t}=\left\{x^{i}, E_{L}\right\}, \quad \frac{d y^{\alpha}}{d t}=\left\{y^{\alpha}, E_{L}\right\}
$$

ile verilir. Bunlardan ilkini kullanarak

$$
\begin{gathered}
\frac{d x^{i}}{d t}=\left\{x^{i}, E_{L}\right\}=\left\{x^{i}, \frac{\partial L}{\partial y^{\alpha}} y^{\alpha}-L\right\} \\
\frac{d x^{i}}{d t}=\left(a_{\mathcal{A}}\right)_{\alpha}^{i} y^{\alpha}
\end{gathered}
$$

ikincisini kullanarak ise,

$$
\begin{gathered}
\frac{d}{d t}\left(\frac{\partial L}{\partial y^{\alpha}}\right)=\left\{\frac{\partial L}{\partial y^{\alpha}}, E_{L}\right\} \\
\frac{d}{d t}\left(\frac{\partial L}{\partial y^{\alpha}}\right)=\left(a_{\mathcal{A}}\right)_{\alpha}^{i} \frac{\partial L}{\partial x^{i}}+C_{\alpha \beta}^{\gamma} \frac{\partial L}{\partial y^{\gamma}} y^{\beta}
\end{gathered}
$$

buluruz $[5,6,19]$. Bulduğumuz bu sonuç Lie cebiroidi üzerindeki hareket denklemleridir. $\mathrm{Bu}$ sonucu aşağıdaki teoremde not edelim.

Teorem 2.2: Lie cebiroidi üzerinde Euler-Lagrange denklemleri mevcuttur. Denklem (21)'de verilen yerel koordinatlar cinsinden

$$
\begin{gathered}
\frac{d x^{i}}{d t}=\left(a_{\mathcal{A}}\right)_{\alpha}^{i} y^{\alpha}, \\
\frac{d}{d t}\left(\frac{\partial L}{\partial y^{\alpha}}\right)=\left(a_{\mathcal{A}}\right)_{\alpha}^{i} \frac{\partial L}{\partial x^{i}}+C_{\alpha \beta}^{\gamma} \frac{\partial L}{\partial y^{\gamma}} y^{\beta}
\end{gathered}
$$

şeklinde bulunur.

Örnek 2.7: $(M \times g)$ etki Lie cebiroidi için Lagrange dinamiğini yazalım. Etki Lie cebiroidinin çapa fonksiyonunun, $m \in M$ ve $\xi \in \mathfrak{g}$ için, 


$$
a: M \times \mathfrak{g} \rightarrow T M, \quad a(m, \xi):=X_{\xi}(m)
$$

şeklinde olduğunu Örnek 2.6'dan hatırlayalım. g Lie cebirinin bazlarını $\left\{e_{a}\right\}$ ile ve $\mathrm{M}$ katmanının koordinatlarını ise $\left(x^{i}\right)$ ile gösterirsek $\xi=\xi^{\alpha} e_{a}$ olarak yazabiliriz. Şimdi bu notasyonlar ile

$$
\begin{gathered}
a_{m}: \mathfrak{g} \rightarrow T_{m} M, \\
a_{m}(\xi)=\left(a_{m}\right)_{\alpha}^{i}\left(\xi^{\alpha}\right)=\left(X_{\xi}(m)\right)^{i} \\
a_{m}^{*}: T_{m}^{*} M \rightarrow \mathfrak{g}^{*}, \\
a_{m}^{*}(\varphi)=\left(a_{m}\right)_{\alpha}^{i} \varphi_{i} e^{\alpha}
\end{gathered}
$$

fonksiyonlarını yazalım. Burada $\left(a_{m}\right)_{\alpha}^{i}$ matris gösterimi ve $\left\{e^{\alpha}\right\},\left\{e_{a}\right\}^{\prime}$ nın dual bazıdır. $(M \times g)$ Lie cebiroidinin kesitlerinin bazlarını

$$
\bar{e}_{\alpha}: M \rightarrow M \times \mathfrak{g}, \quad \bar{e}_{\alpha}(m):=\left(m, e_{a}\right)
$$

ile ifade edebiliriz. Böylece etki Lie cebiroidinin çerçeve işlemi

$$
\begin{aligned}
{\left[\bar{e}_{\alpha}, \bar{e}_{\beta}\right]_{(M \times \mathfrak{g})}(m) } & =\left(m,\left[e_{a}, e_{\beta}\right]_{\mathfrak{g}}\right) \\
& =\left(m, c_{\alpha \beta}^{\gamma} e_{\gamma}\right)
\end{aligned}
$$

şeklinde ifade edilebilir, yine burada $c_{\alpha \beta}^{\gamma}, \mathfrak{g}$ Lie cebirinin yapı sabitidir.

$\mathrm{Bu}$ bilgiler ışı̆̆ında $(M \times \mathfrak{g})$ etki Lie cebiroidi üzerindeki Euler-Lagrange denklemleri,

$$
\begin{gathered}
\dot{x}^{i}=a_{\alpha}^{i} \xi^{\alpha}=\left(X_{\xi}(m)\right)^{i} \\
\frac{d}{d t}\left(\frac{\partial L}{\partial \xi^{\alpha}}\right)=a_{\alpha}^{i} \frac{\partial L}{\partial x^{i}}+c_{\alpha \beta}^{\gamma} \frac{\partial L}{\partial \xi^{\gamma}} \xi^{\beta}
\end{gathered}
$$

şeklinde olur. Koordinatlar olmadan ifade etmek istersek, bu durumda Euler-Lagrange denklemleri

$$
\begin{gathered}
\dot{m}=X_{\xi}(m) \\
\frac{d}{d t}\left(\frac{\delta L}{\delta \xi}\right)=a_{m}^{*}\left(\frac{\delta L}{\delta m}\right)-a d_{\xi}^{*}\left(\frac{\delta L}{\delta \xi}\right)
\end{gathered}
$$

şeklinde hesaplanır ve burada $\delta L / \delta m \in T^{*} M$, $\delta L / \delta \xi \in \mathrm{g}^{*}$ ile verilir.

\section{Lie Cebiroidleri Üzerindeki Eşlenmiş Lagrange Dinamiği}

\subsection{Eşlenmiş Lie grupoidi}

Eşlenmiş Lie grupoidinden bahsetmeden önce Lie grupoidi etkilerini verelim. $\mathcal{G}, B$ tabanı üzerinde bir Lie grupoidi ve $f: P \rightarrow B$ de bir $P$ katmanindan grupoidinin tabanı $B$ 'ye düzgün bir fonksiyon olsun. Kısmi çarpım uzayı

$$
P * \mathcal{G}:=\{(p, g) \in P \times \mathcal{G} \mid f(p)=\alpha(g)\},
$$

için, bir

$$
\triangleleft: P * \mathcal{G} \rightarrow P, \quad(p, g) \mapsto p \triangleleft g
$$

düzgün fonksiyonu her $(p, g) \in P * \mathcal{G},\left(g, g^{\prime}\right) \in \mathcal{G} * \mathcal{G}$, ve $p \in P$ için

$$
\begin{aligned}
& f(p \triangleleft g)=\beta(g), \\
& (p \triangleleft g) \triangleleft g^{\prime}=p \triangleleft\left(g g^{\prime}\right), \\
& p \triangleleft \overline{f(p)}=p,
\end{aligned}
$$

özellikleri sağlıyorsa bu fonksiyona $\mathcal{G}^{\prime}$ nin $f$ üzerine (sağ) etkisi denir. Bir Lie grupoidinin bir düzgün fonksiyon üzerine sol etkisi de benzer şekilde tanımlanır, [12, 20].

Böylelikle, bir Lie grupoidinin bir başka Lie grupoidi üzerine etkisini tanımlamaya hazırız. $\mathcal{G}, B$ tabanı üzerinde bir Lie grupoidi, $\mathcal{H}$ de bir $C$ tabanı üzerinde başka bir Lie grupoidi olsun. Şimdi $\mathcal{H}^{\prime}$ nin $\mathcal{G}^{\prime}$ ye sol etkisi $\mathcal{H}^{\prime}$ nin $\alpha: \mathcal{G} \rightarrow B$ hedefi üzerine sol etkisi, $\mathcal{G}^{\prime}$ nin $\mathcal{H}$ 'ye sağ etkisi de $\mathcal{G}$ 'nin $\beta: \mathcal{H} \rightarrow C$ hedef fonksiyonuna sağ etkisi olarak tanımlanır, $[20,21]$.

Şimdi $[13,20]$ takip ederek daha önce Lie gruplar için $[22,23]$ 'da verilmiş olan eşlenmiş Lie gruplar teorisinin Lie grupoidi karşılığını özetleyeceğiz. Başlangıç olarak, $\mathcal{G} \rightrightarrows B$ ve $\mathcal{H} \rightrightarrows B$ aynı bir $B$ tabanı üzerinde iki Lie grupoidi olsunlar, $\mathcal{H}$ de $\mathcal{G}$ üzerine soldan

$$
\triangleright: \mathcal{H} * \mathcal{G} \rightarrow \mathcal{G}, \quad\left(h, g^{\prime}\right) \mapsto h \triangleright g^{\prime}
$$

ile etki etsin, yani her $\left(h, g^{\prime}\right) \in \mathcal{H} * \mathcal{G},\left(h^{\prime}, h\right) \in \mathcal{H} * \mathcal{H}$, ve $h \in \mathcal{H}$ için

$$
\begin{aligned}
& \alpha(h)=\alpha\left(h \triangleright g^{\prime}\right), \\
& \left(h^{\prime} h\right) \triangleright g^{\prime}=h^{\prime} \triangleright\left(h \triangleright g^{\prime}\right) \\
& \text { herhangi bir } h \in \mathcal{H} \text { için } \widetilde{\alpha(h)} \triangleright g^{\prime}=g^{\prime}
\end{aligned}
$$

olsun. Aynı şekilde $\mathcal{G}$ de $\mathcal{H}$ üzerine sağdan

$$
\triangleleft: \mathcal{H} * \mathcal{G} \rightarrow \mathcal{H}, \quad\left(h, g^{\prime}\right) \mapsto h \triangleleft g^{\prime}
$$

ile etki etsin, yani her $\left(h, g^{\prime}\right) \in \mathcal{H} * \mathcal{G},\left(g^{\prime}, g\right) \in \mathcal{G} * \mathcal{G}$, ve $g^{\prime} \in \mathcal{G}$ için

$$
\begin{aligned}
& \beta\left(g^{\prime}\right)=\beta\left(h \triangleleft g^{\prime}\right), \\
& h \triangleleft\left(g^{\prime} g\right)=\left(h \triangleleft g^{\prime}\right) \triangleleft g, \\
& h \triangleleft \widetilde{\beta\left(g^{\prime}\right)}=h,
\end{aligned}
$$

sağlansın. Tüm bunlara ilave olarak, eğer her $\left(h, g^{\prime}\right) \in$ $\mathcal{H} * \mathcal{G},\left(g^{\prime}, g\right) \in \mathcal{G} * \mathcal{G}$, ve $\left(h^{\prime}, h\right) \in \mathcal{H} * \mathcal{H}$ için

$$
\begin{aligned}
& \beta\left(h \triangleright g^{\prime}\right)=\alpha\left(h \triangleleft g^{\prime}\right), \\
& h \triangleright\left(g^{\prime} g\right)=\left(h \triangleright g^{\prime}\right)\left(\left(h \triangleleft g^{\prime}\right) \triangleright g\right), \\
& \left(h^{\prime} h\right) \triangleleft g^{\prime}=\left(h^{\prime} \triangleleft\left(h \triangleright g^{\prime}\right)\right)\left(h \triangleleft g^{\prime}\right),
\end{aligned}
$$

sağlanırsa $(\mathcal{G}, \mathcal{H})$ 'ye eșlenmiş Lie grupoidi çifti denilir.

Bu durumda 


$$
\begin{gathered}
\mathcal{G} \bowtie \mathcal{H}:=\mathcal{G} * \mathcal{H} \\
=\{(g, h) \in \mathcal{G} \times \mathcal{H} \mid \beta(g)=\alpha(h)\}
\end{gathered}
$$

olmak üzere kısmi çarpım uzayı

$$
\begin{gathered}
(\mathcal{G} \bowtie \mathcal{H}) *(\mathcal{G} \bowtie \mathcal{H}) \\
:=\left\{\left((g, h),\left(g^{\prime}, h^{\prime}\right)\right) \in(\mathcal{G} \bowtie \mathcal{H}) \times(\mathcal{G} \bowtie \mathcal{H}):\right. \\
\left.\beta(h)=\alpha\left(g^{\prime}\right)\right\},
\end{gathered}
$$

üzerinde tanımlanan

$$
\begin{gathered}
(\mathcal{G} \bowtie \mathcal{H}) *(\mathcal{G} \bowtie \mathcal{H}) \rightarrow(\mathcal{G} \bowtie \mathcal{H}), \\
\left((g, h),\left(g^{\prime}, h^{\prime}\right)\right) \mapsto\left(g\left(h \triangleright g^{\prime}\right),\left(h \triangleleft g^{\prime}\right) h^{\prime}\right)
\end{gathered}
$$

marifetiyle bir Lie grupoidi olur. Bu Lie grupoidi ise eşlenmiş Lie grupoidi olarak anılır. Eşlenmiş Lie grupoidi $\mathcal{G} \bowtie \mathcal{H}^{\prime}$ nin kaynak ve hedef fonksiyonları sirasiyla

$$
\begin{array}{lll}
\alpha: \mathcal{G} \bowtie \mathcal{H} \rightarrow B, & (g, h) \mapsto \alpha(g), \\
\beta: \mathcal{G} \bowtie \mathcal{H} \rightarrow B, & (g, h) \mapsto \beta(h),
\end{array}
$$

olarak tanımlanır. Gömme fonksiyonu ise

$$
\varepsilon: B \rightarrow \mathcal{G} \bowtie \mathcal{H}, \quad b \mapsto(\tilde{b}, \tilde{b})
$$

olarak verilir.

Eşlenmiş Lie grupoidi $\mathcal{G} \bowtie \mathcal{H}$ ile kendisini oluşturan $\mathcal{G}$ ve $\mathcal{H}$ Lie grupoidleri arasındaki ilişki [20, Teo. 2.10]'de verilmiş olup, burada aşağıdaki önermede hatırlatılmaktadır.

Teorem 3.1: $(\mathcal{G}, \mathcal{H})$ Lie grupoidi çiftinin bir eşlenmiş Lie grupoidi çifti olması için gerek ve yeter şartlar

- $\mathcal{G} * \mathcal{H}$ katmanının bir Lie grupoidi yapısına sahip olmass,

- $g \mapsto(g, \widetilde{\beta(g)})$ ile verilen $\mathcal{G} \rightarrow \mathcal{G} * \mathcal{H}$ fonksiyonu ile $h \mapsto(\overline{\alpha(h)}, h)$ şeklinde tanımlanan $\mathcal{H} \rightarrow \mathcal{G} *$ $\mathcal{H}$ fonksiyonunun Lie groupoidi homomorfizmaları olmaları, -çarpma fonksiyonu $((g, \widetilde{\beta(g)}),(\widetilde{\alpha(h)}, h)) \mapsto$ $(g, h) \in \mathcal{G} * \mathcal{H}$ 'nin bir difeomorfizma olmasıdir.

Örnek 3.2: Bir etki grupoidi $\mathcal{G}:=M \times G$ ve bir ikili grupoidi $\mathcal{H}:=M \times M$ verilsin. Aşağıdaki kümeyi göz önüne alalım.

$$
\begin{gathered}
\mathcal{H} * \mathcal{G}=(M \times M) *(M \times G) \\
=\left\{\left(m^{\prime}, m ; m, g\right) \in(M \times M) \times(M \times G)\right\}
\end{gathered}
$$

$\mathcal{G}=M \times G$ grupoidinin $\mathcal{H}=M \times M$ üzerine sol etkisi

$$
\begin{gathered}
\triangleright:(M \times M) *(M \times G) \longrightarrow(M \times G), \\
\left(m^{\prime}, m\right) \triangleright(m, g):=\left(m^{\prime}, g\right)
\end{gathered}
$$

ve sağ etkisi

$$
\begin{gathered}
\triangleleft:(M \times M) *(M \times G) \rightarrow(M \times M), \\
\left(m^{\prime}, m\right) \triangleleft(m, g):=\left(m^{\prime} g, m g\right)
\end{gathered}
$$

(i)-(ix) ile verilen koşulları sağlar. Böylece küme

$$
\begin{gathered}
\mathcal{G} * \mathcal{H}=(M \times G) *(M \times M) \\
=\left\{\left(m, g ; m g, m^{\prime}\right) \in(M \times G) \times(M \times M)\right\}
\end{gathered}
$$

$M$ baz katmanı üzerinde bir eşlenmiş Lie grupoidi $\mathcal{G} \bowtie \mathcal{H}=(M \times G) \bowtie(M \times M)$ olur. Kaynak ve hedef fonksiyonları ile gömme fonksiyonu

$$
\begin{gathered}
\alpha:(M \times G) \bowtie(M \times M) \rightarrow M, \\
\left(m, g ; m g, m^{\prime}\right) \mapsto m, \\
\beta:(M \times G) \bowtie(M \times M) \rightarrow M, \\
\left(m, g ; m g, m^{\prime}\right) \mapsto m^{\prime}, \\
\varepsilon: M \rightarrow(M \times G) \bowtie(M \times M), \\
m \mapsto(m, e ; m, m)
\end{gathered}
$$

olarak verilir. Ayrıca, çarpım uzayı üzerinde

$$
\begin{gathered}
((M \times G) \bowtie(M \times M)) \\
*((M \times G) \bowtie(M \times M)) \\
:=\left\{\left(m, g ; m g, m^{\prime}\right),\left(m^{\prime}, h ; m^{\prime} h, n\right):\right. \\
\left.m^{\prime}, m, n \in M \text { ve } g, h \in G\right\}
\end{gathered}
$$

kısmi çarpımı,

$$
\begin{gathered}
\left(m, g ; m g, m^{\prime}\right) *\left(m^{\prime}, h ; m^{\prime} h, n\right) \\
=\left((m, g)\left(\left(m g, m^{\prime}\right) \triangleright\left(m^{\prime}, h\right)\right) ;\right. \\
\left.\left(\left(m g, m^{\prime}\right) \triangleleft\left(m^{\prime}, h\right)\right)\left(m^{\prime} h, n\right)\right) \\
=\left((m, g)(m g, h) ;\left(m g h, m^{\prime} h\right)\left(m^{\prime} h, n\right)\right. \\
=(m, g h ; m g h, n)
\end{gathered}
$$

olur. Buna göre örneğin tersini alma işlemi

$$
(m, g ; m g, n)^{-1}=\left(n, g^{-1} ; n g^{-1}, m\right)
$$

olarak yazılır $[11,13]$.

Eşlenmiş Lie gupoidi $(M \times G) \bowtie(M \times M)$, aşikar grupoidi $M \times G \times M$ ile ilişkisi

$$
\begin{gathered}
\phi: M \times G \times M \rightarrow(M \times G) \bowtie(M \times M), \\
(m, g, n) \mapsto(m, g ; m g, n),
\end{gathered}
$$

şeklinde tanımlanır [13].

\subsection{Eşlenmiş Lie cebiroidi}

$[13,24,25]$ 'den bir Lie cebiroidinin bir vektör demeti üzerine olan etkisini hatırlayalım. $\mathrm{Bu}$ amaçla, $\left(\mathcal{A}, \tau_{\mathcal{A}}, M, a_{\mathcal{A}},[\bullet, \bullet]_{\mathcal{A}}\right)$ bir Lie cebiroid, ve $(E, \pi, M) \mathrm{de}$ aynı $M$ tabanı üzerinde bir vektör demeti olsun. $\left(\mathcal{A}, \tau_{\mathcal{A}}, M\right)^{\prime} \operatorname{nin}(E, \pi, M)^{\prime}$ ye sol etkisi her $X, \tilde{X} \in \Gamma(\mathcal{A})$, $Y \in \Gamma(E)$, ve $f \in C^{\infty}(M)$ için 


$$
\begin{aligned}
& \rho_{f X}(Y)=f \rho_{X}(Y), \\
& \rho_{X}(f Y)=f \rho_{X}(Y)+\left(a_{\mathcal{A}}(X) f\right) Y, \\
& \rho_{[X, \tilde{X}]_{\mathcal{A}}}(Y)=\rho_{X}\left(\rho_{\tilde{X}}(Y)\right)-\rho_{\tilde{X}}\left(\rho_{X}(Y)\right),
\end{aligned}
$$

özelliklerini sağlayan iki-lineer bir

$$
\begin{gathered}
\rho: \Gamma(\mathcal{A}) \times \Gamma(E) \longrightarrow \Gamma(E), \\
(X, s) \mapsto \rho_{X}(s)=\rho(X, s)
\end{gathered}
$$

fonksiyonu olarak tanımlanır. Bir Lie cebiroidinin bir vektör demeti üzerine sağ etkisi de benzer şekilde tanımlanır.

$\mathcal{A}$ ve $\mathcal{B}$ aynı bir $M$ katmanı üzerinde iki Lie cebiroidi olsunlar. Eğer vektör demeti direkt toplamı $\mathcal{A} \oplus \mathcal{B}$ de aynı $M$ katmanı üzerinde bir Lie cebiroidi oluyorsa ve $\mathcal{A}$ ile $\mathcal{B}$ de direk toplam Lie cebiroidi $\mathcal{A} \oplus \mathcal{B}^{\prime}$ nin Lie alt-cebiroidi oluyorsa, bu $(\mathcal{A}, \mathcal{B})$ çiftine eșlenmiș Lie cebiroidi çifti denir, [13].

Teorem 3.2: Aynı $M$ katmanı üzerinde tanımlı $\left(\mathcal{A}, \tau_{\mathcal{A}}, M, a_{\mathcal{A}},[\bullet, \bullet]_{\mathcal{A}}\right)$ ve $\left(\mathcal{B}, \tau_{\mathcal{B}}, M, a_{\mathcal{B}},[\bullet, \bullet]_{\mathcal{B}}\right)$ Lie cebiroidlerinin birbirleri üzerine olan

$$
\begin{aligned}
& \rho: \Gamma(\mathcal{B}) \times \Gamma(\mathcal{A}) \rightarrow \Gamma(\mathcal{A}), \\
& \rho^{\prime}: \Gamma(\mathcal{A}) \times \Gamma(\mathcal{B}) \rightarrow \Gamma(\mathcal{B}),
\end{aligned}
$$

etkileri her $X, \tilde{X} \in \Gamma(\mathcal{A})$, ve her $Y, \tilde{Y} \in \Gamma(\mathcal{B})$ için

$$
\begin{gathered}
\cdot \rho_{Y}[X, \tilde{X}]_{\mathcal{A}}=\left[\rho_{Y}(X), \tilde{X}\right]_{\mathcal{A}}+\left[X, \rho_{Y}(\tilde{X})\right]_{\mathcal{A}} \\
-\rho_{\rho^{\prime} X(Y)}(\tilde{X})+\rho_{\rho^{\prime} \tilde{X}(Y)}(X), \\
\text { - } \rho^{\prime}{ }_{X}[Y, \tilde{Y}]_{\mathcal{B}}=\left[\rho^{\prime}{ }_{X}(Y), \tilde{Y}\right]_{\mathcal{B}}+\left[Y, \rho^{\prime}{ }_{X}(\tilde{Y})\right]_{\mathcal{B}}+ \\
-\rho^{\prime}{ }_{\rho_{Y}(X)}(\tilde{Y})+\rho^{\prime}{ }_{\rho_{\tilde{Y}}(X)}(Y), \\
\text { - }\left[a_{\mathcal{B}}(Y), a_{\mathcal{A}}(X)\right]=a_{\mathcal{A}}\left(\rho_{Y}(X)\right)-a_{\mathcal{B}}\left(\rho^{\prime}{ }_{X}(Y)\right),
\end{gathered}
$$

şartlarını sağlarsa direkt toplam vektör demeti $\mathcal{A} \bowtie$ $\mathcal{B}:=\mathcal{A} \oplus \mathcal{B}$ üzerinde bir Lie cebiroidi yapısı tanımlı olur.

Bu Lie cebiroidinin kesitler uzayındaki Lie çerçevesi her $X \in \Gamma(\mathcal{A})$ ve her $Y \in \Gamma(\mathcal{B})$ için

$$
[Y, X]_{\bowtie}=\rho(Y, X)-\rho^{\prime}(X, Y)
$$

ile belirlidir. İşte böyle $(\mathcal{A}, \mathcal{B})$ Lie cebiroidi çiftine eşlenmiş Lie cebiroidi çifti, Lie cebiroidi yapısını haiz vektör demeti $\mathcal{A} \bowtie \mathcal{B}^{\prime}$ ye de eşlenmiş Lie cebiroidi denir.

Eşlenmiş Lie cebiroidi çiftinin yerel koordinatlardaki ifadelerini belirtelim. $\left(\mathcal{A}, \tau_{\mathcal{A}}, M, a_{\mathcal{A}},[\bullet, \bullet]_{\mathcal{A}}\right)$ ve $\left(\mathcal{B}, \tau_{\mathcal{B}}, M, a_{\mathcal{B}},[\bullet, \bullet]_{\mathcal{B}}\right)$, aynı bir $M$ katmanı üzerinde tanımlı iki Lie cebiroidi olsunlar. Yukarıda verilmiş olan gösterilimleri takip ederek; $M$ üzerinde bir yerel koordinat sistemimi yine $\left(x^{i}\right)$ ile, $\mathcal{A}^{\prime}$ nın kesitler uzayı $\Gamma(\mathcal{A})^{\prime}$ 'nın izdüşümsel $C^{\infty}(M)$-modül tabanını $\left\{e_{\alpha}\right\}$ ile, ve benzer şekilde $\mathcal{B}$ 'nin kesitler uzayı $\Gamma(\mathcal{B})$ 'nın izdüşümsel $C^{\infty}(M)$-modül tabanını da $\left\{f_{a}\right\}$ ile gösterelim. Böylece, $\left(a_{\mathcal{A}}\right)_{\alpha}^{i},\left(a_{\mathcal{B}}\right)_{k}^{i}, C_{\beta \gamma}^{\alpha}, C_{a b}^{d} \in C^{\infty}(M)$ olmak üzere

$$
\begin{aligned}
a_{\mathcal{A}}\left(e_{\alpha}\right) & =\left(a_{\mathcal{A}}\right)_{\alpha}^{i} \frac{\partial}{\partial x^{i}}, \quad\left[e_{\beta}, e_{\gamma}\right]_{\mathcal{A}}=C_{\beta \gamma}^{\alpha} e_{\alpha} \\
a_{\mathcal{B}}\left(f_{a}\right) & =\left(a_{\mathcal{B}}\right)_{a}^{i} \frac{\partial}{\partial x^{i}}, \quad\left[f_{b}, f_{d}\right]_{\mathcal{B}}=C_{b d}^{a} f_{a}
\end{aligned}
$$

olup $\mathcal{A}$ üzerinde bir yerel koordinat $\operatorname{sistemi}\left(x^{i}, y^{\alpha}\right)$ ile, $\mathcal{B}$ üzerinde bir yerel koordinat sistemi ise $\left(x^{i}, z^{a}\right)$ ile gösterilebilir. Şimdi (yine aynı $M$ katmanı üzerindeki) eşlenmiş Lie cebiroidi $\mathcal{A} \bowtie \mathcal{B}^{\prime}$ yi $\operatorname{de}(\mathcal{A} \bowtie$ $\left.\mathcal{B}, \tau_{\bowtie}, M, a_{\bowtie},[\bullet, \bullet]_{\bowtie}\right)$ beşlisi ile gösterelim. Böylece,

$$
\begin{gathered}
e_{\alpha} \in \Gamma(\mathcal{A}) \longrightarrow\left(e_{\alpha}, 0\right) \in \Gamma(\mathcal{A} \bowtie \mathcal{B}), \\
f_{a} \in \Gamma(\mathcal{B}) \longrightarrow\left(0, f_{a}\right) \in \Gamma(\mathcal{A} \bowtie \mathcal{B})
\end{gathered}
$$

ışı̆̆ında, eşlenmiş Lie cebiroidinin kesitler uzayı $\Gamma(\mathcal{A} \bowtie \mathcal{B})$ için

$$
\left\{e_{\alpha}, f_{a}\right\}:=\left\{\left(e_{\alpha}, 0\right),\left(0, f_{a}\right)\right\}
$$

bazına varırız. Bununla birlikte, $\mathcal{A} \bowtie \mathcal{B}$ üzerinde bir yerel koordinat sistemini de $\left(x^{i}, \bar{y}^{k}\right):=\left(x^{i}, y^{\alpha}, z^{a}\right)$ olarak düșünebiliriz. Burada, $\bar{y}^{k}$ hem $y^{\alpha}$, hem de $z^{a}$ yerini tutmaktadır. Daha açık bir söyleyişle, $k$ ile gösterilen etiketler $\alpha$ ile gösterilenler üzerinde değerler aldıklarında $\bar{y}^{\alpha}:=y^{\alpha}, a$ ile gösterilenler üzerinde değerler aldıklarında ise $\bar{y}^{a}:=z^{a}$ kabul edilmektedir. Bu sade gösteriliși kullanarak, eșlenmiș Lie cebiroidi $\mathcal{A} \bowtie \mathcal{B}^{\prime}$ nin kesitler uzayı $\Gamma(\mathcal{A} \bowtie \mathcal{B})^{\prime}$ nin bazını kısaca $\left\{\bar{e}_{k}\right\}$, yani $\bar{e}_{\alpha}:=e_{\alpha}$ ve $\bar{e}_{a}:=f_{a}$, şeklinde düşünebiliriz. Dahası, böylece, $\mathcal{A} \bowtie \mathcal{B}$ üzerindeki çapa fonksiyonunu da basitçe

$$
a_{\bowtie}\left(\bar{e}_{k}\right)=\tilde{a}_{k}^{i} \frac{\partial}{\partial x^{i}}
$$

olarak ifade edebiliriz. Burada da, $\tilde{a}_{\alpha}^{i}:=\left(a_{\mathcal{A}}\right)_{\alpha}^{i}$, ve $\tilde{a}_{a}^{i}:=\left(a_{\mathcal{B}}\right)_{a}^{i}$ anlamına gelmektedir. Öte yandan, bu iki Lie cebiroidinin birbirleri üzerine olan etkilerini de

$$
\begin{gathered}
\triangleright: \Gamma(\mathcal{B}) \times \Gamma(\mathcal{A}) \rightarrow \Gamma(\mathcal{A}), \\
\left(f_{d}, e_{\gamma}\right) \mapsto f_{d} \triangleright e_{\gamma}:=C_{d \gamma}^{\alpha} e_{\alpha} \\
\triangleleft: \Gamma(\mathcal{B}) \times \Gamma(\mathcal{A}) \rightarrow \Gamma(\mathcal{B}), \\
\left(f_{d}, e_{\gamma}\right) \mapsto f_{d} \triangleleft e_{\gamma}:=C_{d \gamma}^{a} f_{a}
\end{gathered}
$$

ile gösterelim. Böylece, $\Gamma(\mathcal{A} \bowtie \mathcal{B})$ üzerindeki çerçeve işlemi

$$
\begin{gathered}
{\left[f_{d}, e_{\gamma}\right]_{\bowtie}:=\left[\left(0, f_{d}\right),\left(e_{\gamma}, 0\right)\right]_{\bowtie}} \\
=\left(f_{d} \triangleright e_{\gamma}, f_{d} \triangleleft e_{\gamma}\right)=\left(C_{d \gamma}^{\alpha} e_{\alpha}, C_{d \gamma}^{a} f_{a}\right), \\
{\left[\left(e_{\beta}, 0\right),\left(e_{\gamma}, 0\right)\right]_{\bowtie}=C_{\beta \gamma}^{\alpha}\left(e_{\alpha}, 0\right),} \\
{\left[\left(0, f_{b}\right),\left(0, f_{d}\right)\right]_{\bowtie}=C_{b d}^{a}\left(0, f_{a}\right)}
\end{gathered}
$$

ile tam olarak belirlenir.

Örnek 3.3: Bir g Lie cebirinin bir sol etkisine olanak tanıyan bir $M$ katmanı alalım. $M \times g$ bir etki Lie cebiroidi olmak üzere, $T M \bowtie(M \times \mathfrak{g})$ bir eşlenmiş Lie cebiroidi oluşturur [13]. Bu TM $\bowtie(M \times \mathfrak{g})$ eşlenmiş Lie cebiroidinin çapa fonksiyonu 


$$
\begin{gathered}
a_{\bowtie}=T M \bowtie(M \times g) \rightarrow T M, \\
a_{\bowtie}(X+u)=I d_{T M}(X)+a(u)=X+X_{u}
\end{gathered}
$$

izdüșüm fonksiyonu olarak tanımlanır ve buradaki $a(u)$ ile ifade edilen Örnek 2.6 ile verilen çapa fonksiyonudur. $X, Y \in \Gamma(T M)$ kesitleri ile $u, v: M \rightarrow \mathfrak{g}$ fonksiyonları için, $T M \bowtie(M \times \mathfrak{g})$ Lie cebiroidinin kesitleri üzerinde

$$
\begin{gathered}
{[X+u, Y+v]_{\bowtie}} \\
:=[X, Y]_{J L}+[u, v]_{(M \times \mathrm{g})}+\mathcal{L}_{X}(v)-\mathcal{L}_{Y}(u)
\end{gathered}
$$

şeklinde çerçeve işlemi tanımlanır ve burada $\mathcal{L}_{X}(v)$ ile $X \in \Gamma(T M)$ vektör alanı boyunca bir Lie cebir değerli $v: M \rightarrow \mathfrak{g}$ fonksiyonunun Lie türevini ifade etmektedir [26]. Eşlenmiş Lie cebiroidini oluşturan Lie cebiroidlerinin karşılıklı etkilerini

$$
\begin{aligned}
& \triangleright: \Gamma(M \times \mathfrak{g}) \times \Gamma(T M) \rightarrow \Gamma(T M), \\
&(u, Y) \mapsto u \triangleright \mathrm{Y}=0 \\
& \triangleleft: \Gamma(M \times \mathfrak{g}) \times \Gamma(T M) \rightarrow \Gamma(M \times \mathfrak{g}), \\
&(u, Y) \mapsto u \triangleleft \mathrm{Y}=-\mathcal{L}_{Y}(u)
\end{aligned}
$$

olarak hesapları.

Eşlenmiş Lie grupoidinin Lie cebiroidi: $(\mathcal{G} \bowtie$ $\mathcal{H}, B, \alpha, \beta, \varepsilon)$ bir eşlenmiş Lie grupoidi olsun. Kaynak fonksiyonu $\quad \alpha: \mathcal{G} \bowtie \mathcal{H} \rightarrow B \quad$ ve $\quad(g, h) \in \mathcal{G} \bowtie \mathcal{H}$ noktasinda tanjantı $T_{(g, h)} \alpha: T_{(g, h)}(\mathcal{G} \bowtie \mathcal{H}) \rightarrow T_{\alpha(g, h)} B$ olsun. $\mathcal{G} \bowtie \mathcal{H}$ Lie grupoidi ile ilişkilendirilen Lie cebiroidi iplikleri

$$
\mathcal{A}_{b}(\mathcal{G} \bowtie \mathcal{H}):=\operatorname{ker}_{\varepsilon(b)} \alpha,
$$

şeklindedir. $(\mathcal{A}(\mathcal{G} \bowtie \mathcal{H}), \tau, B)$ vektör demeti olarak tanımlanır. Bir başka deyişle $\mathcal{A}(\mathcal{G} \bowtie \mathcal{H}), \mathcal{G} \bowtie \mathcal{H}$ üzerinde $\alpha: \mathcal{G} \bowtie \mathcal{H} \rightarrow B$ fonksiyonuna göre alınan dikey demetine karşıllk gelir. $\tau: \mathcal{A}(\mathcal{G} \bowtie \mathcal{H}) \rightarrow B$ vektör demetinin kesitlerini $X \in \Gamma(\mathcal{A}(\mathcal{G} \bowtie \mathcal{H}))$ ile gösterelim. Çapa fonksiyonu $a: \mathcal{A}(\mathcal{G} \bowtie \mathcal{H}) \rightarrow T B$ ise

$$
a(X(b))=T_{(\tilde{b}, \tilde{b})} \beta \circ X(b)
$$

ile verilir, burada $T_{(\tilde{b}, \tilde{b})} \beta: T_{(\tilde{b}, \tilde{b})}(\mathcal{G} \bowtie \mathcal{H}) \rightarrow T_{b} B$ ile $\beta: \mathcal{G} \bowtie \mathcal{H} \rightarrow B$ hedef fonksiyonunun $(\tilde{b}, \tilde{b})=\varepsilon(b) \in$ $\mathcal{G} \bowtie \mathcal{H}$ noktasındaki tanjantıdır. $\mathcal{A}(\mathcal{G} \bowtie \mathcal{H}) \rightarrow B$ kesitleri üzerindeki Lie çerçevesi $[\bullet, \bullet]_{\mathcal{A}(\mathcal{G} \bowtie \mathcal{H})}$, grupoidi üzerindeki sol (veya sağ) vektör alanlarının Jacobi-Lie çerçevesi yardımıyla tanımlanır. $\mathcal{A}(\mathcal{G} \bowtie$ $\mathcal{H})$ demetinin kesiti $X \in \Gamma(\mathcal{A}(\mathcal{G} \bowtie \mathcal{H}))$ için, karşılık gelen bir sol değişmez vektör alanı $\overleftarrow{X} \in \Gamma(T(\mathcal{A}(\mathcal{G} \bowtie$ $\mathcal{H})$ )) vardir ve

$$
\overleftarrow{X}(g, h):=T_{(\beta \widetilde{(g, h)}, \beta \widetilde{(g, h)})} \ell_{(g, h)} X(\beta(g, h))
$$

ile tanımlanır. Bu sayede Lie çerçeve işlemi, $X, Y \in$ $\Gamma(\mathcal{A}(\mathcal{G} \bowtie \mathcal{H}))$ kesitleri

$$
[X, Y]_{\mathcal{A}(\mathcal{G} \bowtie \mathcal{H})}(b):=[\overleftarrow{X}, \overleftarrow{Y}](\tilde{b}, \tilde{b})
$$

şeklinde yazılır, burada sağ tarafta verilen Jacobi-Lie çerçeve işlemidir. Öte yandan bir $X \in \Gamma(\mathcal{A}(\mathcal{G} \bowtie \mathcal{H}))$ için, ona karşılık gelen bir sağ değișmez vektör alanı $\vec{X} \in \Gamma(T(\mathcal{A}(\mathcal{G} \bowtie \mathcal{H})))$ ise

$$
\begin{aligned}
\vec{X}(g, h):= & -T_{(\alpha \overline{(g, h)}, \alpha \overline{(g, h)})} r_{(g, h)}{ }^{\circ} \\
& T_{(\alpha \overline{(g, h)}, \alpha \overline{(g, h)})} \operatorname{inv}(X(\alpha(g, h))
\end{aligned}
$$

ile tanımlanır, burada inv: $\mathcal{G} \bowtie \mathcal{H} \rightarrow \mathcal{G} \bowtie \mathcal{H}$ tersine çevirme ve $T_{\alpha \overline{(g, h)}} r_{(g, h)}: T_{\alpha \overline{(g, h)}} \mathcal{G} \bowtie \mathcal{H} \rightarrow T_{(g, h)} \mathcal{G} \bowtie \mathcal{H}$ ise $\varepsilon(\alpha(g, h))=(\alpha \overline{(g, h)}, \alpha \overline{(g, h)})$ noktasinda $(g, h) \in$ $\mathcal{G} \bowtie \mathcal{H}$ ile verilen sağ ötelemenin tanjantıdır. Çerçeve işlemi $X, Y \in \Gamma(\mathcal{A}(\mathcal{G} \bowtie \mathcal{H}))$ kesitleri için

$$
\overrightarrow{[X, Y]}_{\mathcal{A}(\mathcal{G} \bowtie \mathcal{H})}=-[\vec{X}, \vec{Y}],
$$

şeklinde yazılır, daha fazla ayrıntı için bakınız [11]. Bütün bu hesaplar sonucunda aşağıdaki teorem, Teorem 3.2'nin sonucu olarak gelecektir.

Teorem 3.3: $\mathcal{A}(\mathcal{G} \bowtie \mathcal{H})$ ile gösterdiğimiz eşlenmiş Lie grupoidi $\mathcal{G} \bowtie \mathcal{H}$ 'ye karşı gelen Lie cebiroidi, $\mathcal{G}$ Lie groupoidine karşl gelen $\mathcal{A G}$ Lie cebiroidi ve $\mathcal{H}$ Lie groupoidine karşı gelen $\mathcal{A H}$ Lie cebiroidinin eşlenmesi ile esyapilıdır:

$$
\mathcal{A}(\mathcal{G} \bowtie \mathcal{H}) \simeq \mathcal{A G} \bowtie \mathcal{A H}
$$

\subsection{Eşlenmiş Lie cebiroidi üzerindeki Lagrange dinamiği}

$\mathcal{A} \bowtie \mathcal{B}$ üzerinde bir yerel koordinat sistemini de yine $\left(x^{i}, \bar{y}^{k}\right):=\left(x^{i}, y^{\alpha}, z^{a}\right) \quad$ olarak düșünelim ve yukarıdakine benzer bir yol izleyerek eşlenmiş Lie cebiroidi $\mathcal{A} \bowtie \mathcal{B}$ üzerinde verilen bir Lagrange fonksiyonu $L \in C^{\infty}(\mathcal{A} \bowtie \mathcal{B})$ 'nin ürettiği EulerLagrange denklemleri Teorem 2.2'nin bir özel durumu olarak

$$
\begin{gathered}
\frac{d x^{i}}{d t}=\tilde{a}_{k}^{i} \bar{y}^{k}, \\
\frac{d}{d t}\left(\frac{\partial L}{\partial \bar{y}^{k}}\right)=\tilde{a}_{k}^{i} \frac{\partial L}{\partial x^{i}}+C_{k l}^{s} \bar{y}^{l} \frac{\partial L}{\partial \bar{y}^{s}}
\end{gathered}
$$

şeklinde elde edilir. Şimdi yapı eşlenmiş Lie cebiroidinin sabitleri $C_{k l}^{s}$ yerine Denklem (54), (58) ve (59)'de hesap ettiğimiz yapı sabitleri ve etkileri belirleyen etki sabitlerini, çapa fonksiyonu $\tilde{a}_{k}^{i}$ yerinede Denklem (54)'teki yerel gösterimleri alalım. Tüm bu özel gösterimleri denklem sistemimizde yerine yerleştirerek hesap sonucunda eșlenmiş EulerLagrange denklemlerini elde ederiz. Aşağıdaki teorem bu hesabin sonucudur.

Teorem 3.4: $\mathcal{A} \bowtie \mathcal{B}$ eşlenmiş Lie cebiroidi üzerindeki eşlenmiş Euler-Lagrange denklemleri Denklem (54), (58) ve (59)'daki yerel gösterimler ışığında

$$
\frac{d x^{i}}{d t}=\left(a_{\mathcal{A}}\right)_{\alpha}^{i} y^{\alpha}+\left(a_{\mathcal{B}}\right)_{a}^{i} z^{a}
$$




$$
\begin{aligned}
\frac{d}{d t}\left(\frac{\partial L}{\partial y^{\beta}}\right)=\left(a_{\mathcal{A}}\right)_{\beta}^{i} \frac{\partial L}{\partial x^{i}}+C_{\beta \gamma}^{\alpha} y^{\gamma} \frac{\partial L}{\partial y^{\alpha}} \\
+C_{\beta d^{\alpha}}^{\alpha} z^{d} \frac{\partial L}{\partial y^{\alpha}}+C_{\beta d^{a} z^{d} \frac{\partial L}{\partial z^{a}},}^{d t}\left(\frac{\partial L}{\partial z^{b}}\right)=\left(a_{\mathcal{B}}\right)_{b}^{i} \frac{\partial L}{\partial x^{i}}+C_{b d}^{a} z^{d} \frac{\partial L}{\partial z^{a}} \\
+C_{b \gamma}^{\alpha} y^{\gamma} \frac{\partial L}{\partial y^{\alpha}}+C_{b \gamma}^{a} y^{\gamma} \frac{\partial L}{\partial z^{a}}
\end{aligned}
$$

olarak elde edilir.

(72) ile verilen denklem takımının ikinci satırına yoğunlaşalım. Să̆ tarafındaki ilk iki terim $\mathcal{A}$ üzerindeki Euler-Lagrange denklemini verir, üçüncü terim $\mathcal{B}$ 'nin $\mathcal{A}$ 'ya sol etkisinden gelen terimdir ve dördüncü terim ise $\mathcal{A}$ 'nın $\mathcal{B}$ 'ye sağ etkisinden gelen terimdir. Üçüncü satıra baktığımızda ise, benzer şekilde, sağ taraftaki ilk iki terim $\mathcal{B}$ üzerindeki Euler-Lagrange denklemini verir, üçüncü terim $\mathcal{B}$ 'nin $\mathcal{A}$ 'ya sol etkisinden gelen terimdir ve dördüncü terim ise $\mathcal{A}$ 'nın $\mathcal{B}$ 'ye sağ etkisinden gelen terimdir.

Son olarak iki açık problem belirleyerek makalemizi noktalayalım. Yüksek mertebeden Euler-Lagrange denklemlerinin Lie cebiroidleri üzerindeki analizi [27] nolu çalışmada ele alınmıştır. [28] nolu çalışma ise yüksek mertebeden Lagrange sistemleri için eşlenme problemini Lie cebirleri üzerinde çalışmıştır. Bir açık problem olarak Lie cebiroidleri üzerinde yüksek mertebeden Lagrange sistemlerinin eşlenme problemini verebiliriz. İleriki çalışmalarımızda bu konuya değinmeyi planlamaktayı. Hamilton sistemlerinin Lie cebirlerin duali üzerinde eşlenme problemi [29] nolu çalışmamızda vermiştik. Bu çalışmanın daha genel bir ifadesi Lie cebiroidlerinin duali üzerinde mümkündür. Bir diğer açlk problem olarak da ileriki çalışmalarımızı bu noktayı tartışmayı planlamaktayı.

\section{Teşekkür}

$\mathrm{Bu}$ çalışma TÜBITTAK, 117F426 numaralı "Eşlenmiş Lagrange ve Hamilton Sistemleri" isimli projenin bir parçasıdır. Destek için TÜBİTAK'a teşekkür ederiz.

\section{Etik Beyanı}

Bu çalışmada, "Yükseköğretim Kurumları Bilimsel Araştırma ve Yayın Etiği Yönergesi" kapsamında uyulması gerekli tüm kurallara uyulduğunu, bahsi geçen yönergenin "Bilimsel Araştırma ve Yayın Etiğine Aykırı Eylemler" başlığı altında belirtilen eylemlerden hiçbirinin gerçekleștirilmediğini taahhüt ederiz.

\section{Kaynakça}

[1] Abraham, R., Marsden, J. E., Marsden, J. E. 1978. Foundations of mechanics . Reading, Massachusetts: Benjamin/Cummings Publishing Company.
[2] Holm, D. D., Schmah, T., Stoica, C. 2009. Geometric mechanics and symmetry: from finite to infinite dimensions. Vol. 12. Oxford University Press.

[3] Yaremko, Y. 2000. The Tangent Groups of a Lie Group and Gauge Invariance in Lagrangian Dynamics. In Proceedings of Institute of Mathematics of NAS of Ukraine. 30(2), 544-550.

[4] Marsden, J. E., Ratiu, T. S. 1995. Introduction to mechanics and symmetry. Physics Today, 48 (12), 65.

[5] Weinstein, A. 1996. Lagrangian mechanics and groupoids. Fields Institute Proc. AMS, 7, $207-$ 231.

[6] Martínez, E. 2001. Lagrangian mechanics on Lie algebroids. Acta Applicandae Mathematica, 67 (3), 295-320.

[7] Martínez, E. 2009. Lie algebroids and Mechanics. In AIP Conference Proceedings, American Institute of Physics, 1130(1), 3-33.

[8] Ratiu, T., Moerbeke, P. V. 1982. The Lagrange rigid body motion. In Annales de l'institut Fourier. 32(1), 211-234.

[9] Holm, D. D., Marsden, J. E., Ratiu, T. S. 1998. The Euler-Poincaré equations and semidirect products with applications to continuum theories. Advances in Mathematics, 137(1), 1-81.

[10] Esen, O., Sütlü, S. 2017. Lagrangian dynamics on matched pairs. Journal of Geometry and Physics, 111, 142-157.

[11] Esen, O., Sütlü, S. 2021. Discrete dynamical systems over double cross-product Lie groupoids. International Journal of Geometric Methods in Modern Physics. 18(04), 2150057.

[12] Mackenzie, K., Kirill, M., Mackenzie, K. C. 1987. Lie groupoids and Lie algebroids in differential geometry. Cambridge university press.

[13] Mokri, T. 1997. Matched pairs of Lie algebroids. Glasgow Mathematical Journal, 39(2), 167-181.

[14] Pradines, J. 1967. Theorie de Lie pour les groupoides differentiable. CR Acad. Sci. Paris, 264, 245-248.

[15] Crampin, M., Saunders, D. 2016. Cartan geometries and their symmetries: a Lie algebroid approach. Springer.

[16] Mackenzie, K. C., Mackenzie, K. C. 2005. General theory of Lie groupoids and Lie algebroids. Cambridge University Press.

[17] Marrero, J. C., de Diego, D. M., Martínez, E. 2006. Discrete Lagrangian and Hamiltonian mechanics on Lie groupoids. Nonlinearity, 19(6), 1313. 
[18] Iglesias, D., Marrero, J. C., Martín de Diego, D., Martínez, E., Padrón, E. 2007. Reduction of symplectic Lie algebroids by a Lie subalgebroid and a symmetry Lie group. SIGMA. Symmetry, Integrability and Geometry: Methods and Applications, 3, 049.

[19] Cortés, J.,de Leon, M., Marrero, J. C., de Diego, D. M., Martinez, E. 2006. A survey of Lagrangian mechanics and control on Lie algebroids and groupoids. International Journal of Geometric Methods in Modern Physics, 3(03), 509-558.

[20] Mackenzie, K. C. 1992. Double Lie algebroids and second-order geometry, I. Advances in Mathematics, 94(2), 180-239.

[21] Brown, R. 1972. Groupoids as coefficients. Proceedings of the London Mathematical Society, 3(3), 413-426.

[22] Majid, S. 1990. Matched pairs of Lie groups associated to solutions of the Yang-Baxter equations. Pacific Journal of Mathematics, 141(2), 311-332.
[23] Majid, S. 2000. Foundations of quantum group theory. Cambridge university press.

[24] Higgins, P. J., Mackenzie, K. 1990. Algebraic constructions in the category of Lie algebroids. Journal of Algebra, 129(1), 194-230.

[25] Lu, J. H. 1997. Lie algebroids associated to Poisson actions. In Duke Math. J.

[26] Mackenzie, K. C. 1995. Lie algebroids and Lie pseudoalgebras. Bulletin of the London Mathematical Society, 27(2), 97-147.

[27] Martínez, E. 2015. Higher-order variational calculus on Lie algebroids. Journal of Geometric Mechanics, 7(1), 81-108.

[28] Esen, O., Kudeyt, M., Sütlü, S. 2021. Second order Lagrangian dynamics on double cross product groups. Journal of Geometry and Physics, 159, 103934.

[29] Esen, O., Sütlü, S. 2016. Hamiltonian dynamics on matched pairs. International Journal of Geometric Methods in Modern Physics, 13(10), 1650128. 